POLÍTICAS PÚBLICAS, PRÁCTICAS CORPORALES Y

REPRESENTACIONES SOCIALES SOBRE LA VEJEZ. UN ESTUDIO DE

CASOS: EL PLAN NACIONAL DE DEPORTE Y LOS JUEGOS

DEPORTIVOS, BUENOS AIRES, LA PROVINCIA ${ }^{1}$

DÉBORA Di DOMIZIO

Universidad Nacional de La Plata / Argentina dedido18@yahoo.com.ar

\title{
Resumen
}

En este artículo se presentan las ideas de una serie de discursos a partir de los cuales se han venido diseñando e implementando políticas públicas que poseen prácticas corporales (en torno a lo deportivo) con adultos mayores y que construyen una particular representación social de la vejez. En dichas políticas públicas, se detectaron dimensiones sociales a estudiar a partir de las cuales se identificaron rasgos que las categorizaron y nos permitieron poner de relieve ciertos aspectos para la interpretación y análisis de las mismas. Los avances preliminares vinculados a un abordaje cualitativo de investigación no persiguieron el fin de universalizar el conocimiento como verdad única y cerrada, sino de ofrecer un marco de comprensión de ciertos rasgos y características de la realidad estudiada. En los discursos de los programas analizados hay una mirada reduccionista de las prácticas corporales en la vejez, en los cuales se representa a las prácticas deportivas y los hábitos ascéticos como bienes utilitarios para el logro de una buena longevidad y, también, de un buen encauzamiento, dejando de lado las significaciones subjetivas y colectivas que operan en los sujetos que atraviesan por dichos escenarios.

Palabras-clave: Representaciones sociales; Adultos mayores; Políticas públicas.

\section{POLÍTICAS PÚBLICAS, PRÁTICAS CORPORAIS E REPRESENTAÇÕES SOCIAIS SOBRE O ENVELHECIMENTO. UM ESTUDO DE CASO: O PLANO NACIONAL DE ESPORTES E OS JOGOS ESPORTIVOS, A PROVÍNCIA DE BUENOS AIRES}

\section{Resumo}

Neste artigo apresentamos as ideias de uma série de discursos a partir da qual as políticas públicas nas práticas corporais (relacionadas ao esporte) vêm sendo concebidas e implementadas com idosos e que constroem uma representação social particular acerca da velhice. Nessas políticas públicas, foram detectadas as dimensões sociais para o estudo, da qual foram identificados e classificados os traços que nos permitiram destacar alguns aspectos para sua interpretação e análise. Os progressos preliminares relacionados à abordagem qualitativa não buscam universalizar o conhecimento, como verdade única e fechada, mas para fornecer um quadro para a compreensão das nuances e características da realidade estudada. Nos discursos dos programas analisados há uma visão reducionista das práticas corporais na velhice, nos quais as práticas esportivas e os hábitos ascéticos são mostrados como bens utilitários para

\footnotetext{
${ }^{1} \mathrm{El}$ texto que presento a continuación refiere a algunos capítulos y parte de las conclusiones correspondientes a la Tesis realizada para graduarme de Magister en Educación Corporal, denominada Políticas públicas, prácticas corporales y representaciones sociales sobre la vejez. Un estudio de casos.
} 
alcançar uma boa longevidade e também uma boa canalização, deixando de lado o subjetivo e o coletivo em que operam aqueles que estão passando por ditos cenários.

Palavras-chave: Representações sociais; Idosos; Políticas públicas.

\section{PUBLIC POLICIES, BODY PRACTICES AND SOCIAL REPRESENTATIONS ON AGEING. A CASE STUDY: THE NATIONAL SPORTS PLAN AND THE SPORTS GAMES, BUENOS AIRES PROVINCE}

\section{Abstract}

In this paper we present the ideas of a number of speeches from which public policies have been designing and implementing that have embodied body practices (related to sport) with seniors and who build a particular social representation of ageing. In these policies, were detected to study social dimensions from which were identified and categorized traits that allowed us to highlight certain aspects for the interpretation and analysis of them. Preliminary progress is linked to a qualitative approach of research that do not pursue knowledge as universal and only closed truth, but to provide a framework for understanding certain features and characteristics of the studied reality. In the speeches of the programs analyzed there is a reductionist view of the body practices in old age, in which is shown to sports and ascetic habits like utilitarian goods for achieving good longevity and also a good channeling, aside subjective and collective meanings that operate in those who are going through these scenarios.

Key-words: Social representations; Ageing; Public policy.

\section{Introducción}

Las políticas de vejez están asociadas a necesidades concretas de los adultos mayores y también a situaciones socialmente construidas que relatan formas específicas, de acuerdo con las cuales se es viejo y se vive la vejez. El hecho de que la adultez mayor sea la franja etaria más desprotegida se vincula en gran medida con las representaciones sociales instauradas (Argañaraz, 2003).

En ese sentido, en el estudio de la vejez, suele abundar la confusión entre los hechos científicos y las representaciones sociales que circulan en el imaginario popular. En estas últimas se pueden observar dos posiciones opuestas. Por un lado, se puede constatar una amplia variedad de mitos y estereotipos que muestran a los adultos mayores como personas incapaces, involutivas, declinantes, pobres, socialmente desintegradas y con escaso interés por revertir todo ese cuadro. En el otro extremo, se encuentran representaciones colectivas que pugnan por una imagen positiva, exitosa, que refieren a un período de despliegue de potencialidades en el que se alcanza la sabiduría. Desde esta perspectiva, se construye la idea de grupo social dinámico, activo, partícipe y creativo en el marco de la cultura en la cual está inserto.

Mi interés radicó en relevar ciertos discursos acerca de la vejez y el envejecimiento y, en particular, las ideas, los saberes, las imágenes, y los pensamientos acerca de éstos que suponen las políticas públicas "de lo corporal" para indagar en las concepciones que subyacen a éstas. De esta forma, logré particularizar el tema y los objetivos de la investigación. Dentro del ámbito de las 
representaciones sociales sobre la vejez, quería relevar aquellas que se enunciaban en algunos documentos de políticas públicas implementadas en planes, proyectos y programas que incluyen prácticas corporales con adultos mayores en jurisdicción nacional (República Argentina) y provincial (Provincia de Buenos Aires) a partir del año 2008.

Las políticas públicas que están destinadas a la vejez no son neutras, sino que expresan los valores de una sociedad y la forma de concebir y definir el fenómeno social del envejecimiento. Así, me propuse indagar el modo en que ese conjunto de representaciones sociales revelan concepciones de cuerpo, de práctica corporal y de vejez, que se tornan decisivas al momento de planificar políticas públicas para este sector.

En el marco de este trabajo, el campo de las políticas públicas de lo corporal elegidas como objeto de estudio se circunscribe a dos: el Plan Nacional de Deporte y los Juegos Deportivos, Buenos Aires, La Provincia que describiré a continuación.

\section{El Plan Nacional de Deporte depende de la Secretaría de Deporte de la Nación, organismo que desde el año 2008 pasó a formar parte del Ministerio de Desarrollo Social de la Nación.}

La Secretaría de Deporte de la Nación sostiene que el deporte es una excelente oportunidad para la formación integral de toda la población; un medio óptimo para mejorar la salud; una estrategia ideal para generar fuentes laborales en redes sociales continuas, y una herramienta legítima para reducir los riesgos y amenazas sociales de nuestro tiempo, en particular los efectos de la pobreza. El Deporte constituye un agente promotor de la calidad de vida de la población, de salud, de educación y de organización comunitaria. Asimismo, es un factor que impacta fuertemente en la economía y el empleo. ${ }^{2}$ El deporte y la actividad física se convierten en auténticos generadores de redes sociales que contribuyen a garantizar el desarrollo humano y la cohesión social de la ciudadanía, colaborando en la reconstrucción del tejido social y propiciando la organización comunitaria.

El Plan Nacional de Deporte 2008-2012 establece los lineamientos de la Política Deportiva Nacional. Incluye Programas y Proyectos basándose para su diseño e implementación en un modelo político nacional y popular. De sus tres grandes líneas de acción (el Plan de Deporte Social, el Plan de Desarrollo Deportivo y el Plan de Deporte Federado y de Representación Nacional), me detendré en el primero, puesto que cuenta con la particularidad de incluir prácticas corporales con adultos mayores.

Por Deporte Social se entiende la práctica de actividades físicas y deportivas orientada a la población en su conjunto, sin discriminación de edad, sexo, condición física, social, cultural o étnica. El Plan de Deporte Social incluye distintos programas que se proponen trabajar en relación con la

\footnotetext{
${ }^{2}$ Ver sitio en línea de la Secretaría de Deporte del Ministerio de Desarrollo Social de la Nación.
} 
inclusión social, la educación, la salud y la discapacidad. Éstos fueron diseñados respetando el concepto de transversalidad, lo que garantiza los principios de universalidad, integralidad y no-discriminación.

Los programas que se destacan son los "Juegos Nacionales Evita", el programa "Nuestro Club", y el programa "Argentina es Nuestra Cancha". Para este estudio, sólo tomaremos los "Juegos Nacionales Evita" para Adultos Mayores, puesto que es el que más se ajusta a las necesidades de nuestro objetivo general de estudio. Los "Juegos Nacionales Evita"33 son competencias deportivas de carácter inclusivo, participativo y formativo para niños, jóvenes y adultos mayores ${ }^{4}$ de todo el país. Entre los aspectos de su normativa ${ }^{5}$, se destaca la inclusión de la población adulta mayor en un evento de estas dimensiones, lo que constituye un hecho completamente nuevo en las políticas de promoción del deporte. Los Juegos surgen frente a la necesidad de generar espacios de promoción y desarrollo de la actividad física, recreativa y deportiva adaptada a las características de los adultos mayores para motivar cambios en las actitudes y hábitos cotidianos, orientados a mejorar su calidad de vida. A su vez, se buscaba concientizar a toda la población sobre la importancia de la práctica de actividad física. Como en otras ediciones de los Juegos Nacionales Evita, se rescatan la promoción de valores propios de la actividad deportiva como el juego limpio, el trabajo en equipo, el respeto por el otro, la solidaridad, el placer por jugar, con el fin de que los adultos mayores puedan trasladar esas experiencias a su vida diaria.

El objetivo es consolidar una instancia de encuentros deportivos entre adultos mayores en el ámbito nacional, a través de la práctica de aquellos deportes y actividades lúdicas que se practiquen masivamente en todas las provincias. La modalidad del evento es competitiva y está destinada a adultos mayores de 60 años, de ambos sexos, autoválidos y físicamente activos, que habitualmente realizan actividad física y deportiva, con posibilidades de caminar y trasladarse por sus propios medios. Los participantes pueden ser representantes de clubes, organizaciones no gubernamentales, organizaciones comunales, municipales, gremios, centros de jubilados, etc. Las disciplinas centrales son tejo, ajedrez,

\footnotetext{
3 Comenzando en 1947, con la iniciativa de Ramón Carrillo y el empuje definitivo de Eva Perón, estos Juegos fueron concebidos originalmente como un gran programa de salud y una herramienta para la inclusión social, resultando una oportunidad inédita para que miles de niños participen de actividades deportivas organizadas.

Desarrollados más allá de su valor intrínseco, los Juegos Evita no sólo han propiciado la construcción de hábitos, sino también han producido conocimientos propios acerca del estado general de la niñez, de sus familias y de toda la población involucrada. Toda esa información producida a partir de la convocatoria masiva de chicos y chicas, con el fin de participar en competencias deportivas, sirvió para retroalimentar las políticas de salud, de educación y de inclusión social del gobierno nacional. Los Juegos están dirigidos a más de 5.000.000 de participantes de todo el país, por lo que el deporte es nuevamente una poderosa herramienta de convocatoria, de promoción de valores y de construcción de hábitos, con un claro sentido social. Con esta larga historia, a partir del año 2003 los Juegos Evita pasaron a formar parte de la Política de Deporte Social impulsada por la Secretaría de Deporte del Ministerio de Desarrollo Social de la Nación. Dirigido a chicos de 12 a 18 años y a adultos mayores de 60, este campeonato se desarrolla en cuatro instancias: municipal, zonal, provincial y nacional.

${ }^{4}$ Esta categoría se incorpora por primera vez en el año 2008.

${ }^{5}$ Manual de Competencias Adultos Mayores. Juegos Nacionales Evita 2009.
} 
newcom, tenis de mesa, sapo y actividades del área cultura. Asimismo se realizan talleres de actividad física y también talleres de intercambio cultural con juegos tradicionales, alternativos y cooperativos.

\section{Juegos Deportivos “Buenos Aires, La Provincia” (ex Torneos Abuelos Bonaerenses), Secretaría de Deportes, Gobierno de la Provincia de Buenos Aires.}

Los Juegos convocan a todos los Municipios de la Provincia de Buenos Aires para que participen en la organización de actividades para personas de la tercera edad, con el objeto de brindar a este sector la posibilidad de practicar manifestaciones artísticas, deportivas y recreativas. En este sentido, si bien la participación es abierta, se pone especial énfasis en dirigirlos a aquellos que, por pertenecer a sectores socioeconómicos más deprimidos, no tienen la posibilidad de concurrir y participar en actividades que la comunidad ya brinda. Por tal motivo, cumplen un rol importante las organizaciones e instituciones, ya que son las encargadas de convocar, nuclear y representar a los abuelos. Desde esta perspectiva, se afirma que el deporte y el arte nos enseñan el sacrificio, la tenacidad, la perseverancia; nos enseñan a no aflojar nunca, a no darnos por vencido. ${ }^{6}$

Estos Juegos están destinados a personas de ambos sexos residentes en la Provincia de Buenos Aires nacidos entre los años 1939 a $1949^{7}$ inclusive. En función de estos marcos cronológicos se organizan tres categorías de participación: A, B y C.

Se permite la participación de Escuelas de Adultos, Clubes, Centros de Fomento, Centros de Jubilados, Instituciones Barriales, Comercios, Fábricas, Organismos Privados, Asociaciones de Beneficencia, Iglesias, Sindicatos, Gremios, etc., con sede en la Provincia de Buenos Aires. La participación es libre y gratuita y puede ser de forma individual, en parejas o en grupos. Las disciplinas deportivas contempladas son: ajedrez, bochas, caminata, gimnasia, natación, pentatlón, pesca, tejo, y disciplinas catalogadas como juegos tradicionales: canasta, chin chon, damas, domino, escoba de 15, generala, lotería, mus, sapo, taba, truco.

Por otro lado, es necesario aclarar la metodología utilizada. En este sentido, quiero hacer algunas consideraciones sobre el método cualitativo utilizado en esta investigación.

\section{Aspectos metodológicos}

El tema de investigación evidenció la necesidad de implementar una metodología cualitativa que permitiera dar cuenta de la complejidad de la problemática abordada. Respecto del método cualitativo para analizar temas que involucren a los adultos mayores, Fassio (2008: 35) plantea que este tipo de

\footnotetext{
${ }^{6}$ En http://www.juegosdeportivos.gba.gov.ar/index.asp

${ }^{7}$ Esta última fecha se va modificando buscando siempre el marco cronológico de 60 años.
} 
abordaje permite "dar cuenta de la especificidad del proceso de envejecimiento individual desde la concepción teórica del envejecimiento diferencial".

En el abordaje cualitativo se propicia una mayor flexibilidad y provisionalidad. Esto significa que el investigador debe estar dispuesto a reformular su plan de investigación, desandar su camino y realizar los cambios de rumbo necesarios de acuerdo con los hallazgos que se van logrando desde los inicios. Implica, a su vez, que el proyecto no quede definido por completo desde el comienzo, sino que se vaya construyendo en un proceso de ida y vuelta permanente en la medida en que se va avanzando en la investigación.

En las políticas públicas que estudié y que comparé, he detectado dimensiones sociales a estudiar. Estas podrían entenderse como categorías de análisis tal vez arbitrarias, pero que al imprimir una denominación me permitieron poner de relieve ciertos aspectos para la interpretación y el análisis de las mismas. A su vez, cada dimensión englobó la lista de categorías sociales que en este marco denominé "rasgos". El concepto de rasgo lo tomé de Herkovitz quien lo utiliza para designar a la "unidad más pequeña identificable en una cultura dada" (1952: 190). De acuerdo con este enfoque, un rasgo en las políticas públicas que incluyen prácticas corporales para adultos mayores, estaría dado por aquella unidad que se pueda identificar en los planes, programas y proyectos seleccionados, de tal manera que podamos llevar a cabo la comparación de todos los rasgos en los programas elegidos. En otras palabras, podría explicarse que los rasgos son una categoría social, constituyen datos explícitos o referentes empíricos que surgen del análisis de los documentos en los cuales se fundan los programas seleccionados.

Esos hallazgos tienen respuestas provisorias relativas a los contextos en que se produce el relevamiento de datos que abren nuevas preguntas y plantean posibles líneas de especialización, las cuales pueden extenderse en estudios más profundos de la temática en cuestión.

A modo de conclusión, quiero describir algunas de las orientaciones pautadas en los fundamentos teóricos y metodológicos de las políticas públicas de lo corporal (en torno a lo deportivo) descriptas precedentemente.

\section{Conclusiones}

\section{Plan Nacional de Deporte. Ministerio de Desarrollo Social de la Nación Argentina:}

1. Se representan viejos políticamente correctos y saludables, donde se inscribe el plan en una perspectiva tradicional higienista o biologizante que se basa casi exclusivamente en las ciencias vinculadas a la medicina y la biología. Se considera que el deporte es una escuela que moldeará al buen ciudadano - adulto mayor. De esta forma, aparece una perspectiva moralista que busca extender la práctica deportiva a vastos sectores de la población y con ello conformar / configurar la Nación. Se 
destaca la construcción de civilidad que, mediante el concepto de desarrollo humano, permitiría a los individuos ser más aptos para actuar en sociedad y más útiles a ella. Bracht sostiene que se le otorga al deporte un abordaje positivo - funcionalista, donde el deporte colabora con la armonía de la sociedad en la que se inserta. Asociado a ese valor, también se potencia el aspecto educativo del deporte. La relación entre deporte y educación que aquí se sustenta estaría enraizada en las teorías reproductoras, según las cuales la educación se encuentran al servicio de la clase dominante (Bracht 1996: 66). Tanto el deporte como la actividad física ${ }^{8}$ darán repuesta a la cuestión social ${ }^{9}$. Es por ello que mediante el acceso a la práctica sistemática de distintas actividades, se garantiza la integración del individuo a la sociedad, dándole una identidad nacional y restableciendo las redes sociales. La práctica deportiva es más apropiada para desarrollar valores conforme al sistema que valores superadores o cuestionadores del sistema.

2. Una cuestión de derechos, pero sin contemplar la subjetividad: el desarrollo del deporte comunitario es un dato que marca no sólo un acceso masivo a las prácticas deportivas, sino también modificaciones sustantivas respecto del rol del Estado en el deporte comunitario (Rodríguez 2009:102). Se recomienda un enfoque participativo en el sentido de que represente a todos los colectivos de la sociedad y no se limite sólo a algunas clases. Se instala así el deporte como un derecho universal, en el que el Estado, tal como lo proponen distintos organismos, tratados y leyes internacionales a los cuales los países adhieren, está obligado a ofertar prácticas deportivas. Sin embargo, no aparecen referencias que contemplen la dimensión subjetiva de los destinatarios de estas políticas.

3. La inclusión como eje: Si bien se presenta a los Torneos Evita como inclusores, formativos y participativos para todos los adultos mayores del país, la modalidad es competitiva y de ningún modo recreativa. Además, para participar, los adultos mayores deben cumplir con una serie de características relacionadas con su condición de independencia ${ }^{10}$. Entonces, estarían aptos para participar de estos Juegos todas aquellas personas cuyo estado biológico lo permita. Puesto que se requiere de ellos que habitualmente realicen ya no sólo actividad física en general, sino también actividad deportiva en particular. Teniendo en cuenta la modalidad competitiva y la condición de independencia, podría reflexionarse acerca del nivel de inclusión de estos Juegos, ya que los adultos mayores que encuadran en un universo de estas características resulta reducido.

\footnotetext{
8 Hemos decidido usar la expresión "actividad física” en función de mantener concordancia terminológica con el marco teórico de los planes analizados en detrimento de la expresión "prácticas corporales". En los planes analizados, las actividades físicas hacen referencia a un conjunto de configuraciones de movimiento que no pueden ser clasificadas como deportes. No desconocemos las connotaciones biologicistas que se inscriben en esta expresión ni las críticas que ha suscitado su uso en el campo de la Educación Física.

${ }^{9}$ La cuestión social supone la desintegración del tejido social por medio de un mecanismo de desafiliación de los ciudadanos a la estructura social, por lo que quedan excluidos del sistema.

10 El vocablo "independiente" apunta a lo físico-motor; es decir, que pueda moverse solo sin que nadie lo ayude. En cambio, "autonomía" se refiere a la capacidad mental de decidir lo que quiere hacer y lo que no.
} 
4. Se representa a la vejez, como deterioro y prevención: los Juegos Nacionales Evita para Adultos Mayores ponen de manifiesto la importancia de la actividad física desde una perspectiva utilitaria vinculada principalmente con los rendimientos orgánicos, y los beneficios en la promoción y prevención de la salud. Además, incorpora beneficios en la dimensión social de esa práctica por el establecimiento de redes sociales, la participación, la inclusión, la sociabilidad, etc. Por último, regula y ordena un "uso adecuado" y hegemónico del tiempo libre, sin tener en cuenta otras dimensiones de la esfera emocional, afectos, deseos, gustos o vinculada con el placer de los participantes.

La actividad física que se propone tiene la particularidad de ser adaptada a las características del grupo etario. En relación a ello, considero que se trataría de un modelo que todavía persiste en quienes ven al adulto mayor como un "paciente" o "discapacitado" que tiene deficiencias a ser tratadas, basados en el paradigma biomédico, el cual desde siempre vinculó vejez con enfermedad, asociado al discurso de la Gerontología tradicional (exalta degeneraciones, deterioro, pérdidas). Se espera que esas actividades físicas adaptadas generen en estos sujetos hábitos no nocivos para su salud ya que moldearán sus costumbres a través de la práctica.

\section{Juegos Deportivos, Buenos Aires, La Provincia (ex Torneos Abuelos Bonaerenses), Secretaría de Deportes, Gobierno de la Provincia de Buenos Aires.}

1. La normalización: si bien se trata de jurisdicciones distintas, los fundamentos de esta política pública pueden tener concordancia con los lineamientos propuestos en el Plan Nacional de Deporte Social, ya que se representa a un adulto mayor normalizado. Para estos sujetos, hay una concepción del deporte utilitarista, higienista y moralista. Pedráz (1997) explica que se observa una perspectiva individualista que tiende a depositar en los sujetos la responsabilidad de los éxitos y fracasos personales en términos de adaptación - adecuación social.

2. El deporte, la cultura y la educación como las claves de la inclusión. Para los grupos que son representados como vulnerables, es decir, las personas con capacidades diferentes y los adultos mayores o "abuelos" (en palabras del Gobernador), el deporte, la cultura y la educación son planteados como claves para su inclusión. .

Aquí se presenta una construcción de la adultez mayor en un sentido familiar y ameno, a causa del desconocimiento de que la abuelidad constituye una categoría social que se construye y de que no todas las personas que participan son abuelos. Es decir, que se mantiene la idea tradicional de abuelo, la cual ostenta un carácter anacrónico a principios del siglo XXI.

3. La edad como categoría: coincidentemente con los Torneos Evita, se utiliza la edad cronológica o de calendario (60 años) conformando tres categorías de edad para la participación e inclusión en los Juegos. Dicho marco de referencia es un dato vacío, que no representa la condición sociocultural del 
sujeto. Es esencialmente biológica y se manifiesta en niveles de trastorno funcional. En este sentido, hay que tener en cuenta que el envejecimiento conlleva cambios en la posición del sujeto en la sociedad, debido a las responsabilidades y privilegios que dependen de la edad cronológica (Huenchuan Navarro 1999: 13).

4. Los saberes perdidos. En relación con lo planteado en líneas más arriba, la definición de vejez se realiza en términos cronológicos. Esto podría inducir a pensarla como una realidad homogénea, desconociendo la condición de seres individuales y sociales con contextos históricos, políticos, económicos, culturales y de género determinantes en su construcción histórica. Estas formas de rotular la vejez denotan falta de conocimiento en la temática gerontológica, la cual reclama una fundamentación teórica y metodológica en el área mayor a la que efectivamente se tiene que puede resultar escasa. Se observa un énfasis discursivo a favor de involucrarse con la autodisciplina y las normas de comportamiento socialmente aprobadas, con las cuales también pueden normalizarse y encausarse los adultos mayores.

A modo de síntesis, y teniendo en cuenta lo plasmado en ambos programas que incluyen prácticas corporales para adultos mayores que tienen como base al deporte, puedo afirmar que se representa a la vejez desde una posición teórica que enfatiza el uso de las prácticas corporales en adultos mayores para cambiar estilos de vida, lograr la salud e integrarse a la sociedad. En estos programas, las prácticas corporales y los hábitos ascéticos son bienes utilitarios para lograr una buena longevidad (salud) y un buen encauzamiento. Esta salud es entendida desde el punto de vista biológico y no como construcción social.

En efecto, la vejez y el envejecimiento son representados mediante nociones e ideas naturalizadas, pero sin los fundamentos de una Gerontología Social Crítica. Existe una mirada reduccionista de las prácticas corporales en la vejez asociadas al paradigma positivista (medicina tradicional) y el uso de categorías biologizadas que actúan como preconceptos.

Se pondera la representación de un sujeto adulto mayor con un estilo de vida activo, a quien se llama a participar. Se le otorga al deporte un carácter inclusivo, que busca ofrecer a los adultos mayores su inserción social, pero, al mismo tiempo, sólo se invita a participar a los adultos mayores que gozan de total autonomía e independencia física.

Para terminar, creo importante señalar que es necesario indagar en las concepciones de la vejez que se encuentran en otras políticas públicas, ya que distintas miradas y puntos de vista sobre el envejecimiento son posibles.

Por un lado, podemos afirmar, junto con Martines y Mezzadri (2009, 2010) y con Bracht (1996), que las políticas públicas destinadas al sector deportivo son entendidas como un instrumento del Estado para infundir la disciplina, la autoridad y el control. Pero, desde otra perspectiva, cabe agregar 
que estas políticas públicas para el sector deportivo pueden comportar significados e intereses muy diferentes para los sujetos que las practican, de tal modo que la planificación de las mismas esté realmente vinculada a las necesidades y deseos de los sujetos.

Quizá sea necesario contemplar y tener en cuenta las experiencias singulares y creativas de cada sujeto adulto mayor a la hora de diseñar políticas públicas destinadas a este sector.

\section{Bibliografía}

Araya Umaña, S. (2002). "Las representaciones sociales: Ejes teóricos para su discusión". En Cuadernos de Ciencias Sociales, $n^{\circ}$ 127. Sede Académica Costa Rica. Facultad Latinoamericana de Ciencias Sociales.

Blessmann, E. (2007). "A sociabilidade e a ludicidade nos jogos esportivos adaptados para idosos" En Stigger M .P.; Gonzalez F. J; Silveira R. (org.). Esporte na cidade: estudos etnográficos sobre sociabilidades esportivas em espaços urbanos. Porto Alegre: Editora de la Universidade Federal do Rio Grande do Sul, pp. 147-160.

Bracht, V. (1996). Educación Física y Aprendizaje Social. Córdoba: Editorial Vélez Sarsfield.

Fassio, A. (2008). "Investigación gerontológica: discusiones sobre el análisis cualitativo y la triangulación metodológica". En Molina, S. (comp.). Estrategias comunitarias en el trabajo con adultos mayores. Buenos Aires. Red de Editoriales Universitarias, pp. 47-57.

Fraga, A. B. (2005). Exercício da informação: governo dos corpos no mercado da vida ativa. Tesis de Doctorado. Universidad Federal do Rio Grande do Sul. Facultad de Educación. Porto Alegre.

Herskovits, M. J. (1968). El hombre y sus obras, La ciencia de la antropología cultural. México: Fondo de Cultura Económica.

Huenchuan Navarro, S. (1999). "De Objetos de Protección a Sujetos de Derecho: Trayectoria y Lecciones de las Políticas de Vejez en Europa y Estados Unidos". En Revista de Trabajo Social Perspectivas: Notas sobre Intervención y Acción Social, No 8, Universidad Católica Cardenal Raúl Silva Henríquez, Santiago de Chile.

Iacub R. (2006). Erótica y vejez: perspectivas de occidente. Buenos Aires: Paidós.

Mariluz, G. (2008). Política, Estado y Vejez. Las políticas sociales para la tercera Edad en Argentina desde el Virreynato del Río de la Plata hasta el año 2000. Tesis de maestría inédita. Universidad de Buenos Aires. Facultad de Ciencias Sociales. Buenos Aires.

Martines, I. C. y Marinho Mezzadri F. (2007) "Os modelos de análise sociológica e a elaboração de políticas públicas: a necessidade de novos olhares para o esporte". En Anais do XV Congresso Brasileiro de Ciências do Esporte [e] II Congresso Internacional de Ciências do Esporte / Colégio Brasileiro de Ciências do Esporte. Recife: CBCE, 2007. 
Rodríguez, M.G. (2009). "Peronismo y deporte (1945-1955): entre el experimento y lo conocido". En Crisorio R. y Giles, M. (dirs). Estudios críticos de Educación Física. Colección textos básicos. La Plata: Al Margen, pp. 93-113.

Vendruscolo, R. y Marconcin, P. (2006). "Um estudo dos programas públicos para idosos de alguns municípios paranaenses: a atividade física, esportiva e de lazer em foco". En: Mezzadri, F.; Cavichiolli F; Souza, D. Esporte e Lazer: subsídios para o desenvolvimento e a gestão de políticas públicas. San Pablo: Fontoura, pp. 75-92.

Yuni, J. (2000). "El mito del eterno retorno. Educación, subjetividad y adultos mayores" en Duschatzky, S. et al. (comps). Tutelados y asistidos. Programas sociales, políticas públicas y subjetividad. Buenos Aires: Paidós, pp 187-237. 